\title{
Triboelectrostatic Separation for Recycling of Seaweed-Drying Net Frame Plastic Wastes
}

\author{
Chul-Hyun Park and Ho-Seok Jeon* \\ Korea Institute of Geoscience and Mineral Resources, Daejeon 305-350, Korea
}

In this research, on the basis of the control of electrostatic charge, a laboratory-scale triboelectrostatic separation for separating polypropylene (PP) and acrylonitrile-butadiene-styrene (ABS) in seaweed-drying net frame plastic wastes has been carried out. High density polyethylene (HDPE) in a charger material selection tests using a vertical-reciprocation system was found to be the most effective materials for a tribo-charger in the separation of PP, and ABS. In lab-scale triboelectrostatic separation tests using the HDPE pipeline charger, the charge-tomass ratio $(\mathrm{nC} / \mathrm{g})$ of the mixed $\mathrm{PP}$ and $\mathrm{ABS}$ increased when the air velocity was adjusted to over $10 \mathrm{~m} / \mathrm{s}$. The optimum splitter position estimated by Newton's separation efficiency $\left(\eta_{\mathrm{n}}\right)$ was a position $+4 \mathrm{~cm}$ from the central. Furthermore, under the conditions of over $385 \mathrm{kV} / \mathrm{m}$ electric field and less than $30 \%$ relative humidity, a PP grade of $99.70 \%$ and a recovery of $92.30 \%$ could be obtained. [doi:10.2320/matertrans.MRA2008268]

(Received August 12, 2008; Accepted December 12, 2008; Published February 25, 2009)

Keywords: seaweed-drying, recycling, plastic wastes, tribocharging, electrostatic separation

\section{Introduction}

Net frame plastic wastes which are used as its supports in a dry-processing of seaweeds are generated approximately 20 thousand ton per year from west-south coast area in Korea. ${ }^{1)}$ The seaweed-drying net frame plastics are composed of polyamide (PA), polypropylene (PP) and acrylonitrile-butadiene-styrene (ABS). PA made of yarn is rejected by air classification, but PP and ABS having a similar specific gravity are difficult to separate by a conventional method and so are mostly land-filled or accumulated in the field, causing economic loss as well as environmental contamination. ${ }^{2)}$

Presently, the worldwide production of plastics has reached a level of 150 million tons per year, ${ }^{3)}$ and its consumption keeps growing at a rate of approximately 5\% annually. About 4 million tons of plastic wastes are generated every year in Korea, but only less than $30 \%$ of them are recycled. ${ }^{45)}$ Plastic wastes including PVC can release hazardous substances such as hydrogen chloride gas and dioxins during disposal procedures and its additives can be released by leaching and contact transference. ${ }^{6,7)}$ Also, mixed plastic wastes are difficult to recycle because of their inferior characteristics. In this regard, the disposal of plastic wastes has become an important issue all over the world due to their increasing volume and the decreasing landfill capacity for disposal. ${ }^{8,9)}$

The EU's member states have enforced the environmental regulation such as "Restriction on the use of Certain Hazardous Substances" (RoHS, 2006), "Waste Electrical and Electronic Equipment Directive" (WEEE, 2005) and "End of Life Vehicles Regulations" (ELV, 2002). ${ }^{10-12)}$ Also, the enforcement of "Act on the Promotion of Saving and Recycling of Resources" (2003) and "21c Frontier-Resource Recycling R\&D Projects" (2001) in Korea have restricted the landfill and incineration of plastic wastes and have promoted the recycling of plastic wastes. ${ }^{4,5)}$ Thus, a development of

*Corresponding author, E-mail: hsjeon@kigam.re.kr separation technology for recycling plastic wastes with upswing of oil and raw-material prices has been demanded.

Electrostatic phenomenon is utilized in numerous technical applications such as electro-photography, electrostatic copy and printing techniques, electrostatic filtration and coloring. This method has originally been used for processing valuable minerals like titanium, gold, iron ore as well as coal and fly ash, and nowadays, it is used to recover valuable materials from wastes. ${ }^{11-13)}$

Opposite polarity charge between two particles occurs after particle-particle and particle-charger's surface tribocharging due to their work function. ${ }^{14,15)}$ Charge transfer mechanism in tribocharging can be explained by three models: electron transfer, ion transfer and material transfer. ${ }^{16,17)}$ It has been reported that an electron transfer among them mainly affects the contact charging between materials. ${ }^{16-18)}$ In the process of tribocharging, two materials that are brought into contact or collision can undergo charge transfer according to their work function difference until the point at which their Fermi levels equalize. The final charge will actually be the outcome of two processes: the charge transfer occurs during the contact between materials, and the charge backflow also occurs as they are separated. ${ }^{15,18)}$

This separation method can improve the separation efficiency by establishing a triboelectric series and developing a charger material and charger type ${ }^{19-23)}$ such as cyclone, vibrating feeder, fluidized bed, inclined rotating drum, honeycomb and spiral tube charger, and appropriate handling technique for the various charging factors. ${ }^{24-26)}$

This work aims at developing a reliable and relatively inexpensive technology for the effective triboelectrostatic separation of PP and ABS, based on the control of the charge polarity and charge-to-mass ratio of those particles. Particularly, our study is focused on estimating process variables such as charger material, air velocity, electrode potential, splitter position and relative humidity that affect the separation efficiency of plastics. Therefore, we designed and assembled a laboratory-scale triboelectrostatic separator 


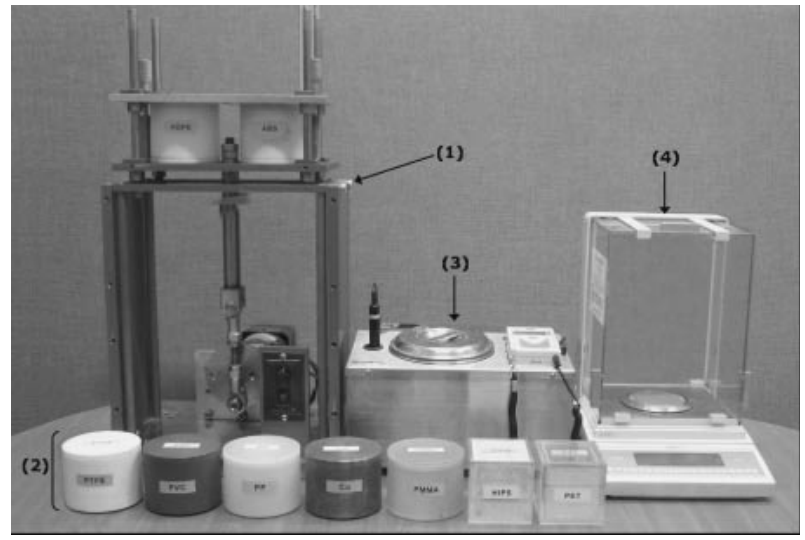

Fig. 1 A vertical-reciprocation charger set and peripheral measurement equipments. ((1) vertical-reciprocation charger, (2) charging bottles, (3) Faraday cage, (4) electric balance)

unit, including a fluidized bed pipe line tribocharger, and evaluated various variables.

\section{Experimental}

\subsection{Materials}

Seaweed-drying net frame plastics used in this study were obtained from a local plant (Econics, Co., Ltd.) and composed of $85 \%$ polypropylene (PP), $10 \%$ acrylonitrilebutadiene-styrene (ABS) and 5\% polyamide (PA). These were shredded with a cutting mill (pulverisette 19, Fritsch $\mathrm{GmbH}$, Germany) and sieved into a fraction of $-4.0+$ $0.5 \mathrm{~mm}$. The initial charge of the particles charged by a cutter was neutralized with a discharger (Kasuga Denki Inc., Japan) and finally, the neutralized particles were fed into triboelectrostatic separator.

\subsection{Equipments}

Two types of a vertical reciprocation charger set and triboelectrostatic separation system were employed for main experiments. Figure 1 shows a charger-set consisting of a vertical reciprocator and charging bottles. A tribocharger material in triboelectrostatic separation system significantly affects the charge polarity and charge-to-mass ratio of the plastics to be separated. Thus, this equipment has been used for charger material selection. The bottles were made of different materials such as polytetrafluoroethylene (PTFE), polyvinyl chloride (PVC), polypropylene (PP), high density polyethylene (HDPE), polyethylene terephthalate (PET), high impact polystylene (HIPS), acrylonitrile butadiene styrene (ABS) or polymethyl methacrylate (PMMA). Inner and outer wall of each bottle were made of the same material. The charger was designed to charge the particles into a charging bottle by reciprocation.

Figure 2 shows a schematic diagram of a triboelectrostatic separator and peripheral equipment used in this work. It consists of a feeding zone (6, 7, 8, and 9), charging zone (1), separation zone (2 and 3 ) and collecting zone (4).

\subsection{Methods}

Figure 3 shows a flowchart for the separation of mixed net frame plastics. They were shredded under $-4 \mathrm{~mm}$ size using

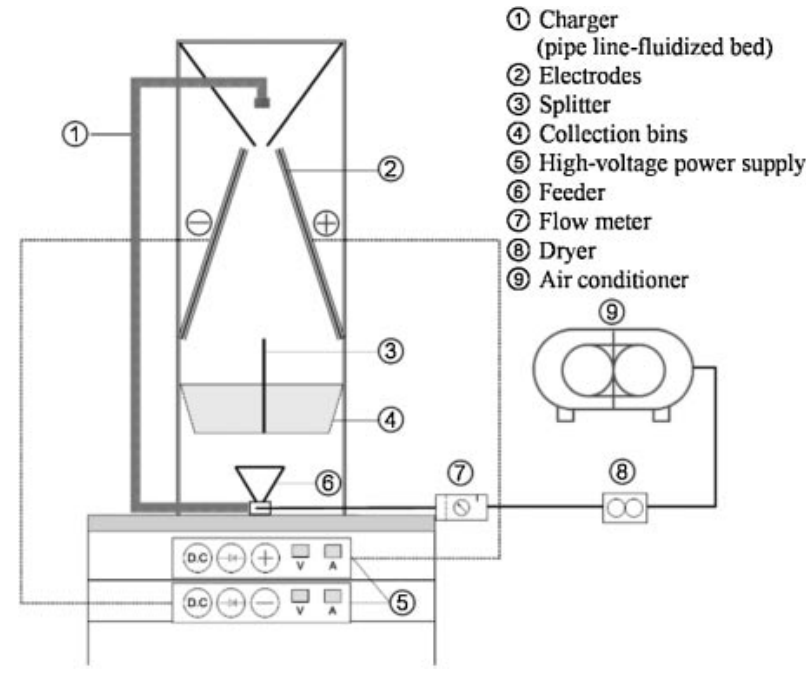

Fig. 2 Schematic diagram of triboelectrostatic separator unit.

a cutting mill (pulverizette 19, Fritsch GmbH, Germany), and then a representative fraction of the plastics was sieved as a fraction of $-4.0+0.5 \mathrm{~mm}$. PA made of yarn was rejected under $10 \mathrm{~m}^{3} / \mathrm{h}$ air rates by zigzag air classification, and then the content of PP and ABS was $89.5 \%$ and $10.5 \%$, respectively. The charge of the plastic particles (PP and ABS) induced by the cutting mill or air classifier was neutralized with a discharger. In charger material selection tests, $20 \mathrm{~g}$ each of PP and ABS was put into different charging bottles, and the charge polarity and charge-to-mass ratio of plastics in the charger were compared at $30 \%$ relative humidity and 3 min retention time.

In triboelectrostatic separation tests for separation of PP and $\mathrm{ABS}$, the mixture was fed into a pipe line charger made of HDPE with flowing air. Then, PP was negatively charged and ABS positively charged by the charger. The negatively charged PP particles were deflected to the positive electrode under the influence of the electric field between the electrodes and the positively charged ABS to the negative electrode.

The Faraday cage used for a charge measure of the particles was a model KQ-1400 (Kasuga Denki Inc., Japan), and its measurement ranged from 1 to $9999 \mathrm{nC}$. The weight of individual plastics was measured with an electronic balance (BP 2100s, Sartorius). Hence, the charge density or specific charge of plastic particles was determined as a charge-tomass ratio $(\mathrm{nC} / \mathrm{g})$. In addition, the data shown in figures are the average values from the tests repeated at least three times.

\subsection{Estimation of degree of the separation}

Newton efficiency is also well known and often used in the field of mineral processing. Thus, the above two items for estimating different process variables in triboelectrostatic separation of plastics were used, and in addition, assessment of a function as splitter position among different variables utilized Newton's separation efficiency ${ }^{25)}\left(\eta_{\mathrm{n}}\right)$ together with grade and recovery. A main target material in this work is PP in net frame plastics and PP grade is the content of PP material in the product fraction, and its recovery and $\eta_{\mathrm{n}}$ in the following: 


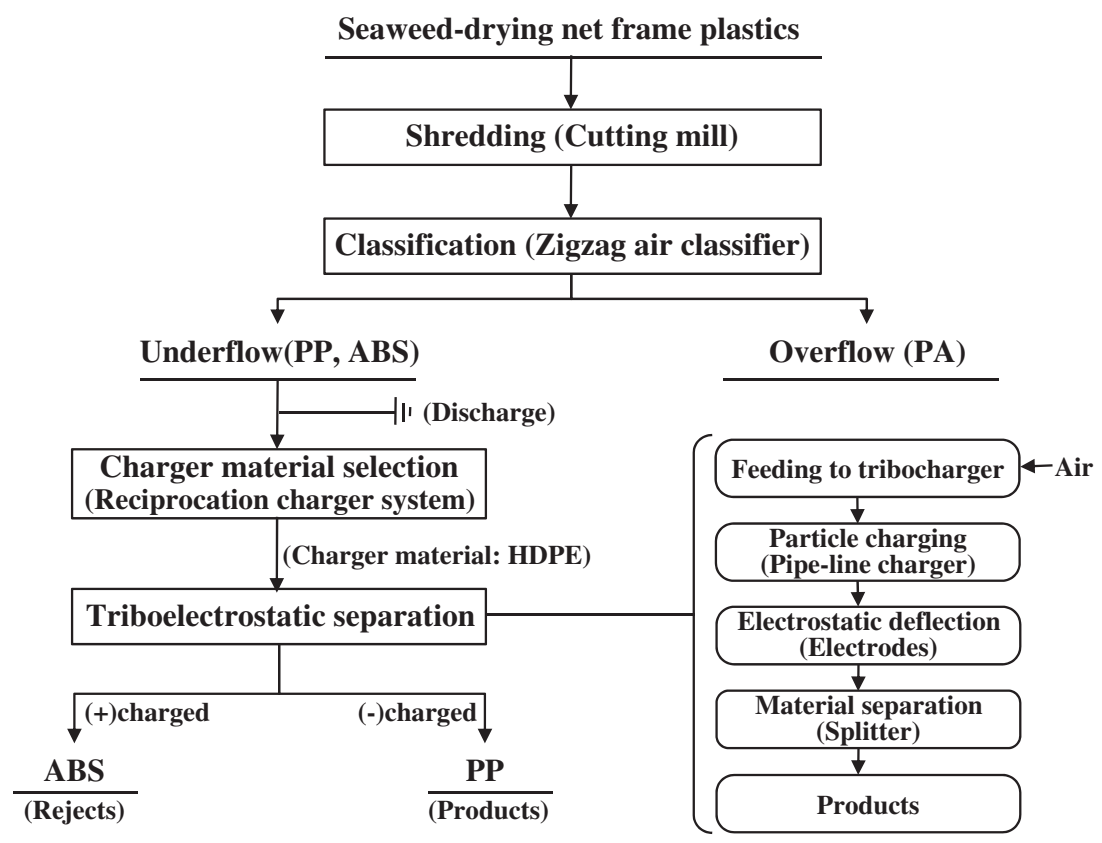

Fig. 3 Flowchart of the recycling process for seaweed-drying net frame plastics.

$$
\begin{aligned}
\mathrm{R}_{\mathrm{c}} & =\frac{c \mathrm{C}}{f F}=\frac{(f-t) c}{(c-t) f} \\
\mathrm{R}_{\mathrm{t}} & =\frac{T(1-t)}{F(1-f)}=\frac{(f-c)(1-t)}{(t-c)(1-t)} \\
\eta_{\mathrm{n}} & =\mathrm{R}_{\mathrm{c}}-\left(1-\mathrm{R}_{\mathrm{t}}\right)=\frac{(f-t)(c-t)}{f(1-f)(c-t)}
\end{aligned}
$$

Where, $R_{c}$ is the recovery of PP in the product fraction; $R_{t}$ is the recovery of ABS in reject fraction; $\eta_{\mathrm{n}}$ is the Newton's efficiency (Tsunekawa, 2005), and $f$ : the content of target material in the feed; $c$ : the content of target material in the product fraction; $t$ : the content of target material in the reject fraction; $F$ : mass of the feed; $\mathrm{C}$ : mass of the product fraction; $T$ : mass of the reject fraction.

\section{Results and Discussion}

\subsection{Charger material selection: charge polarity and charge density of PP and ABS}

Tribocharging is generally controlled by the relative work functions of the particles and inner wall in charger, and there a charger material is an important parameter in selective charging of ABS and PP. The results of the charge polarity and charge density of ABS and PP determined by different charger materials (bottles) is shown in Fig. 4. Experiments were performed at $120 \mathrm{~s}$ retention time, 250 frequency and $30 \%$ relative humidity. ABS $(4.08 \mathrm{eV})$ and $\mathrm{PP}(5.04 \mathrm{eV})$ in chargers made of PTFE, PVC (5.32 eV), PP, ABS or PMMA $(3.72 \mathrm{eV})$ were charged as either the same polarity or hardly charged, but ABS in those materials made of HDPE $(4.72 \mathrm{eV})$, PET $(4.42 \mathrm{eV})$ or HIPS $(4.31 \mathrm{eV})$ were charged positively, and PP negatively. ${ }^{1,4)}$

In case of the former, both $\mathrm{ABS}$ and $\mathrm{PP}$ were charged positively in the charger made of PTFE, PVC, PP because their work function is lower than that of PTFE or PVC, whereas they were charged negatively in that of ABS, PMMA. In case of the latter, the charge polarity of ABS and

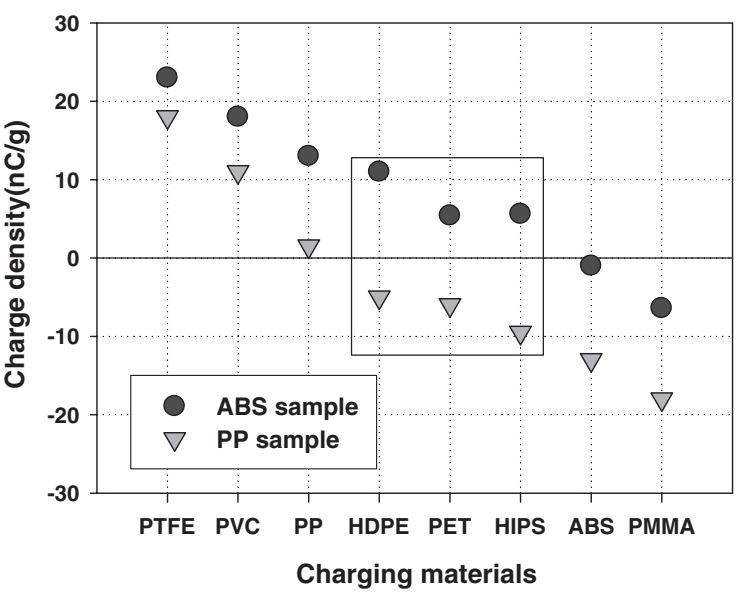

Fig. 4 Charge polarity \& charge density of ABS and PP on charger materials (bottles). (120 s retention time, 250/min frequency and $30 \%$ relative humidity)

PP in a HDPE, PET or HIPS charger is attributed to their work functions laid between ABS and PP from the triboelectric series.4,24) Especially, the difference of charge density between ABS and PP was found to be the maximum with HDPE charger compared to those with PET and HIPS. For example, the charge densities of $\mathrm{ABS}$ and $\mathrm{PP}$ with the HDPE charger were +11 and $-5.5 \mathrm{nC} / \mathrm{g}$ and so the charge density difference was $16.5 \mathrm{nC} / \mathrm{g}$. Thus, the pipe line charger made of HDPE was selected in subsequent triboelectrostatic separation.

The results, as mentioned above, could be explained by triboelectric series or the difference between the work function of the various plastics. ${ }^{23,24)}$ The triboelectric series which represents the degree of the work function values of materials is compared in Table 1. Polymers including halogen elements tend to be negatively charged more than the others because electron affinity is large. Our reference 
Table 1 Comparison of five published triboelectric series. (Diaz and FelixNavarro, 2004; Park, et al., 2007))

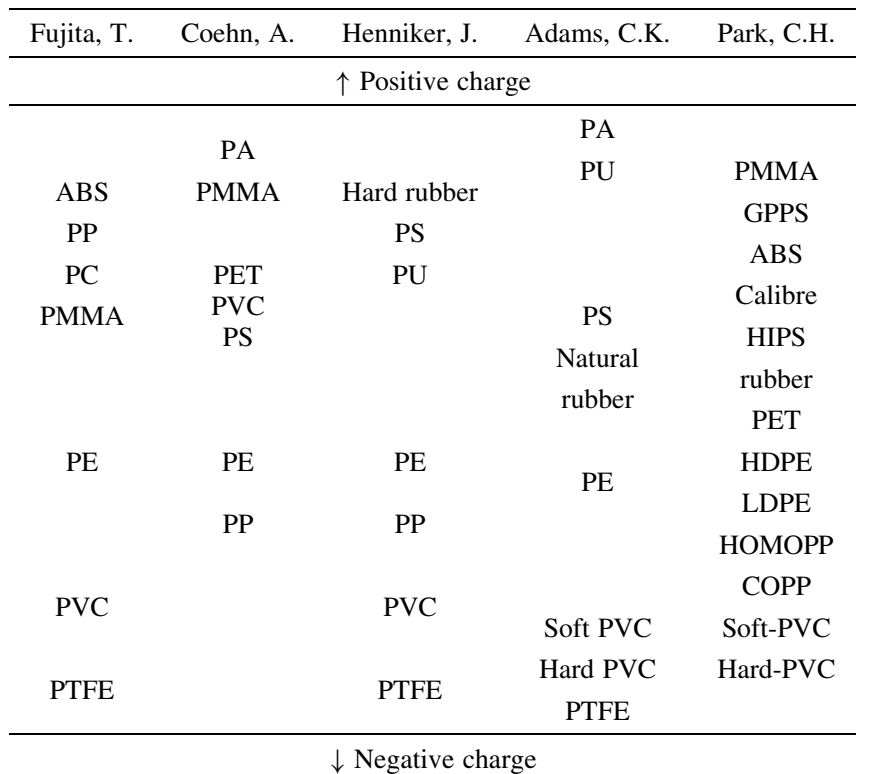

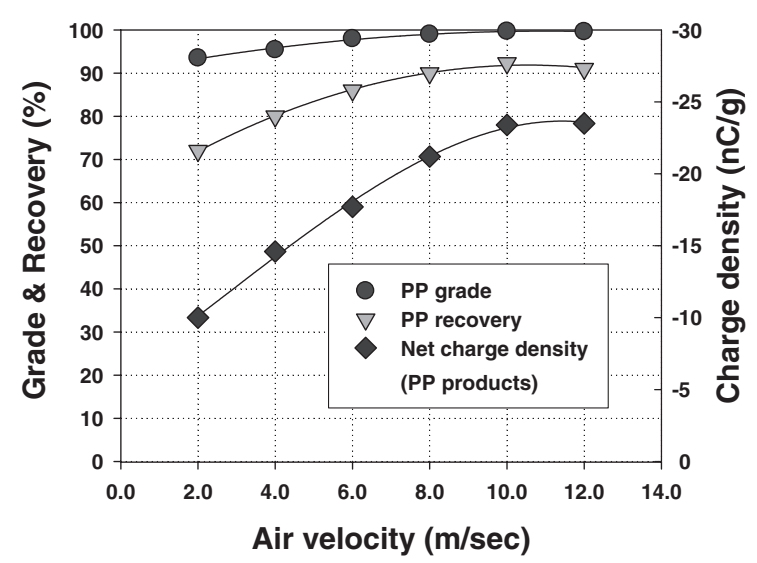

Fig. 5 Effect of air velocity on separation of PP and ABS in triboelectrostatic separation unit. (HDPE charger, 30\% relative humidity, $385 \mathrm{kV} / \mathrm{m}$ electric field and $+4 \mathrm{~cm}$ splitter position)

(Park, 2007) is similar with Adams' study and HDPE is laid between ABS and PP, and some differences between researchers seem like the effect of additives such as plasticizers, resin stabilizers and pigments in plastics.

\subsection{Effect of air velocity}

Tribocharging of mixed plastics occurs when they are fed into a pipe line charger with an air. Thus, the surface chargeto-mass ratio and separation of plastics can be improved by adjusting the air velocity. The effect of air velocity on the net-charge-to-mass ratio and degree of separation of PP from ABS in a triboelectrostatic separation unit is shown in Fig. 5. Experiments were performed at HDPE charger, 30\% relative humidity, $385 \mathrm{kV} / \mathrm{m}$ electric field and $+4 \mathrm{~cm}$ splitter position.

A net charge density of PP increased as the air velocity increased, having a saturation charge value of $23.4 \mathrm{nC} / \mathrm{g}$ at a $10 \mathrm{~m} / \mathrm{s}$ air velocity. This indicates that the charge of the particles depends on air velocity. An increase of air velocity

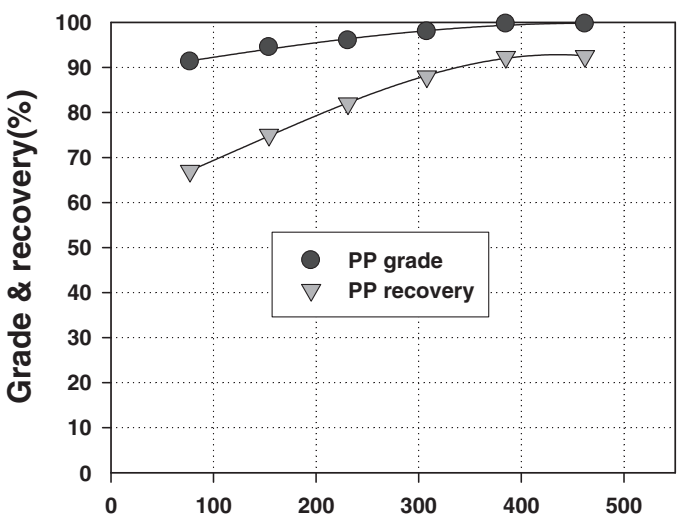

Electric field $(\mathrm{kV} / \mathrm{m})$ between upper electrodes

Fig. 6 Effect of relative humidiy on separation of PP and ABS in triboelectrostatic separation unit. (HDPE charger, $10 \mathrm{~m} / \mathrm{s}$ air velocity, $385 \mathrm{kV} / \mathrm{m}$ electric field and $+4 \mathrm{~cm}$ splitter position)

in a fluidized bed may cause an increase in the impact force and frequency of contact or collision between particle to particle, or particle to surface. Then, the increase of the charge-to-mass ratio is due to an increase of electron transfer between materials with increasing exterior energy by air. ${ }^{25,27)}$

In the case of the separation, grade and recovery of PP reached a maximum at $10 \mathrm{~m} / \mathrm{s}$ air velocity. It is plausible that the falling velocity of the particles increases with an increase in the air velocity. Thus, some particles were moved toward the opposite collection bin after strongly colliding on the splitter or electrode, and then both the PP grade and recovery decreased a little. Therefore, $10 \mathrm{~m} / \mathrm{s}$ of air velocity was determined to be optimum for achieving a relatively high netcharge-to-mass ratio and separation efficiency of plastics in the triboelectrostatic separation.

\subsection{Effect of electric field}

When the charged particles enter the electric field formed between the electrodes, they are subjected to a combination of electrostatic, gravity and drag forces. ${ }^{15,20)}$ Then, the charged particles will be deflected primarily by electrostatic force and partly by gravity and drag force toward negative and positive electrodes.

The effect of electric field on the degree of separation of PP from ABS in a triboelectrostatic separation unit is shown in Fig. 6. Experiments were performed at HDPE charger, $10 \mathrm{~m} /$ $\mathrm{s}$ air velocity, $30 \%$ relative humidity and $+4 \mathrm{~cm}$ splitter position. Both the PP grade and recovery increased as the electric field increased. At a $77 \mathrm{kV} / \mathrm{m}$ electric field, the PP grade and recovery were $91.30 \%$ and $67.1 \%$, respectively. It appears that the electric field of the $77 \mathrm{kV} / \mathrm{m}$ is not strong enough to pull the charged particles toward the electrodes. At a $385 \mathrm{kV} / \mathrm{m}$, a $99.70 \%$ PP grade and $92.30 \%$ PP recovery were successfully obtained. The results show that the PP grade and recovery increased with increasing the electric field.

\subsection{Effect of relative humidity}

The effect of relative humidity on the degree of separation of PP from ABS in a triboelectrostatic separation unit is shown in Fig. 7. Experiments were performed with the 


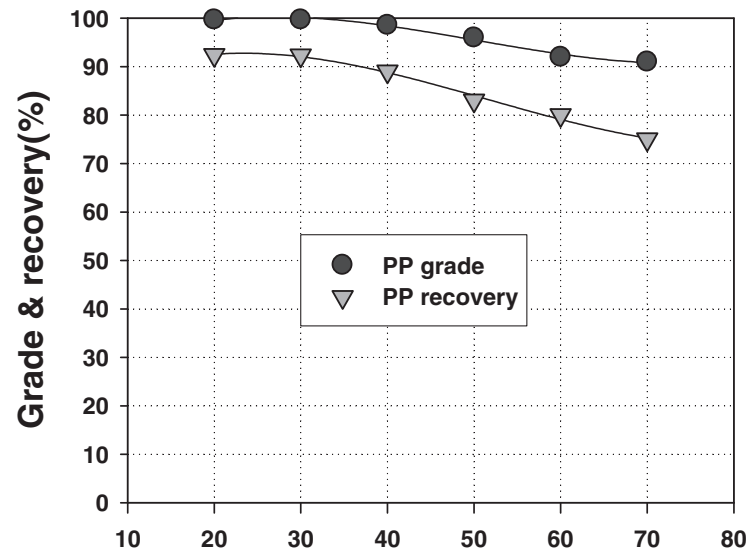

Relative humidity(\%)

Fig. 7 Effect of electric field on separation of PP and ABS in triboelectrostatic separation unit. (HDPE charger, $10 \mathrm{~m} / \mathrm{s}$ air velocity, $30 \%$ relative humidity and $+4 \mathrm{~cm}$ splitter position)

relative humidity range $20-70 \%$ at HDPE charger, $10 \mathrm{~m} / \mathrm{s}$ air velocity, $385 \mathrm{kV} / \mathrm{m}$ electric field and $+4 \mathrm{~cm}$ splitter position.

The acceptable separation of PP could be obtained as long as the relative humidity was less than $30 \%$. However, the degree of separation of PP decreased as the relative humidity increased. With increasing relative humidity, water films are formed on the plastic surface, ${ }^{28)}$ and then it disturbs the surface polarization between particles when contacting or colliding with particles. In addition, moisture in the atmosphere decreases the surface charge of pre-charged particles due to discharging its electron. ${ }^{24)}$

\subsection{Effect of splitter position}

The effect of splitter position on the separation efficiency of PP from ABS in a triboelectrostatic separation unit is shown in Fig. 8. Experiments were performed at a constant HDPE charger, $10 \mathrm{~m} / \mathrm{s}$ air velocity, $385 \mathrm{kV} / \mathrm{m}$ electric field and $30 \%$ the relative humidity. The PP grade increased as the splitter position was moved from the negative electrode to the positive one, and the PP recovery increased as moved from the positive electrode to the negative one.

The falling position of particles can vary, depending on charging factors such as work function (or triboelectric series), charger material, mixture ratio, air velocity and relative humidity under a definite gravity force, drag force and electrostatic force $(30,38-48)$. PP particles which have a high negative charge-to-mass ratio are strongly deflected to the positive electrode, but some PP particles which have a neutral charge-to-mass ratio fall freely. Such behavior of the particles deteriorated the separation efficiency.

Therefore, the optimum splitter position was estimated by Newton's separation efficiency $\left(\eta_{\mathrm{n}}\right)$. A curve of $\eta_{\mathrm{n}}$ increased considerably, depending on PP grade from $-8 \mathrm{~cm}$ splitter position to $+2 \mathrm{~cm}$, and then it decreased by degrees with a decrease of PP recovery from the point of $+4 \mathrm{~cm}$. As mentioned above (Methods item), the content of PP and ABS was each $89.5 \%$ and $10.5 \%$ after PA was rejected by zigzag air classification. In regard to an increase of $\eta_{\mathrm{n}}$ curve in an interval of $-8 \mathrm{~cm}$ to $+2 \mathrm{~cm}$, we think that PP grade affect

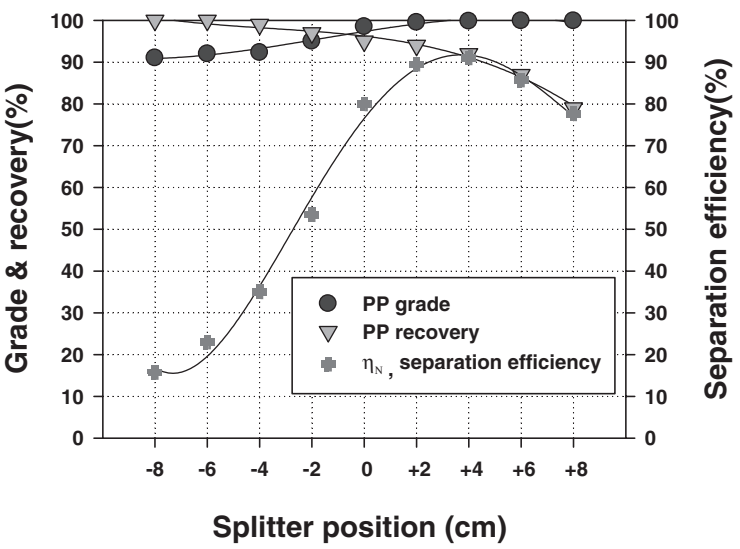

Fig. 8 Effect of splitter position on separation of PP and ABS in triboelectrostatic separation unit. (HDPE charger, $10 \mathrm{~m} / \mathrm{s}$ air velocity, $30 \%$ relative humidity and $385 \mathrm{kV} / \mathrm{m}$ electric field)

considerably $\eta_{\mathrm{n}}$ although PP grade increases some by degrees from $89.5 \%$ to over $99 \%$ because PP grade in feed is high. Considering the results, the best separation efficiency $\left(\eta_{\mathrm{n}}\right.$ : $91 \%$ ) of overall result was achieved at a splitter position $+4 \mathrm{~cm}$ from the center toward the positive electrode, which seems to be the optimum position. At this time, a PP grade of $99.70 \%$ and a recovery of $92.30 \%$ were obtained at the splitter position.

\section{Summary}

In this study, the triboelectrostatic separation of PP and ABS in seaweed-drying net frame plastic wastes has been performed. Firstly, ABS and PP in charger material selection tests using a vertical-reciprocation system were charged positively and negatively, respectively in HDPE, PET, or HIPS charger. The difference of charge density of the ABS and PP was higher in the charger made of HDPE among them. These results demonstrate that the charge polarity and charge density of plastics depend on work function or triboelectric series.

In triboelectrostatic separation using a HDPE pipe line charger, the charge density of PP considerably depended on the air velocity $(23.4 \mathrm{nC} / \mathrm{g}$ at $10 \mathrm{~m} / \mathrm{s})$, and the optimal conditions for electric field and relative humidity affecting the separation efficiency of PP were over $385 \mathrm{kV} / \mathrm{m}$, and $30 \%$. Furthermore, the optimum splitter position estimated by Newton's separation efficiency $\left(\eta_{\mathrm{n}}\right)$ was a position $4 \mathrm{~cm}$ from the center toward the positive electrode. At this time, PP grade considerably affects $\eta_{\mathrm{n}}$ from $89.5 \%$ to over $99 \%$. Under these conditions, a PP grade of $99.70 \%$ and a recovery of $92.30 \%$ were successfully obtained. The results of the separation tests show that triboelectrostatic separation is a reliable, simple and relatively inexpensive technology to recycle plastic wastes.

\section{Acknowledgments}

The authors would like to express special thanks to the Resource Recycling R\&D Center, a 21st Century Frontier R\&D program of the Ministry of Education, Science \& Technology of Korea for financial support. 


\section{REFERENCES}

1) C. H. Park: Doctoral thesis (hanyang university, 2007) 137-138.

2) H. S. Jeon, C. H. Park, S. H. Baek and C. Delgermaa: Digests of the 2006 Spring Meeting and 27th Conference, (2006) 119-123.

3) B. T. Simoneit, P. Medeiros and B. Didyk: Environ. Sci. Technol. 37 (2003) 652-656.

4) C. H. Park, H. S. Jeon and J. K. Park: Korea Inst. Geosci. Mater. Resour. 43 (2006) 560-569.

5) H. S. Jeon, C. H. Park, B. G. Kim and J. K. Park: J. Korean Inst. Resour. Recycl. 15 (2006) 28-36.

6) M. Y. Wey, L. J. Yu and S. I. Jou: J. Hazard. Mater. 60 (1998) 259-270.

7) V. Gente, F. L. Marca, F. Lucci and P. Massacci: Waste. Manage. 23 (2003) 951-958.

8) L. Amelia, C. Hill and J. C. Powell: Resour. Conserv. Recycl. 17 (1996) 75-96.

9) L. W. Reid: J. Hazard. Mater. 47 (1996) 295-302.

10) P. Cusack and T. Perrett: Plastics Additives Compound. 8 (2006) 46-49.

11) J. Cui and E. Forssberg: J. Hazard. Mater. B 99 (2003) 243-263.

12) M. Nourreddine: J. Hazard. Mater. A 139 (2007) 481-490.
13) T. X. Li, J. C. Ban, J. M. Hower and K. Stencel: J. Electrostat. 47 (1999) 133-142.

14) M. Lungu: Miner. Eng. 17 (2004) 69-75.

15) E. G. Kelly and D. J. Sottiswood: Miner. Eng. 2 (1989) 33-46.

16) J. Lowell and A. C. Rose-Innes: Adv. Phys. 29 (1980) 947-1023.

17) J. Lowell: J. Appl. Phys. 12 (1979) 1541.

18) A. R. Akande and J. Lowell: J. Electrost. 16 (1985) 147-156.

19) M. J. Pearse and T. J. Hickey: Resour. Recovery Conserv. 3 (1978) 179-190.

20) I. I. Inculet, G. S. P. Castle and J. D. Brown: Part. Sci. Technol. 16 (1998) 91-100.

21) Y. Matsushita, N. Mori and T. Sometani: Electr. Eng. Jpn. 127 (1999) 33-40.

22) L. Dascalescu: J. Electrost. 63 (2005) 705-710.

23) A. Iuga: J. Electrost. 63 (2005) 937-942.

24) A. F. Diaza: J. Electrost. 62 (2004) 277-290.

25) E. Nemeth, V. Albrecht and G. Schubert: J. Electrost. 58 (2002) 3-16.

26) M. Tsunekawa, B. Naoi, S. Ogawa, K. Hori, N. Hiroyoshi, M. Ito and T. Hirajima: Int. J. Miner. Process. 76 (2005) 67-74

27) J. Lowell: J. Electrost. 26 (1991) 261-273.

28) M. Lungu: Miner. Eng. 17 (2004) 69-75. 